\title{
Développement d'un Examen Clinique Objectif Structuré pour évaluer les compétences des internes en urologie
}

\author{
Louis Sibert", Paul Grand'Maison**, Bernard Charlin ${ }^{\star \star *}$, Philippe Gise*
}

\begin{abstract}
Résumé Contexte : Bien que l'Examen Clinique Objectif Structuré (ECOS) soit considéré comme le meilleur instrument pour l'évaluation de la compétence clinique, l'expérience de ce type d'examen est encore limitée en France. But: Evaluer la faisabilité et l'acceptabilité d'un ECOS destiné aux internes en Urologie Matériel et Méthodes : 20 internes d'urologie ont passé un circuit de 5 stations de 10 minutes chacune avec patients simulés. La sélection des problèmes a été effectuée en fonction des objectifs d'apprentissage des programmes d'enseignement de l'urologie. Un questionnaire écrit avec échelle de Likert a été utilisé pour évaluer l'acceptabilité de l'ECOS. Les performances des internes juniors et seniors ont été comparées avec un test $t$ de Student. Résultats: La performance des internes seniors a été globalement supérieure à celle des juniors, mais sans différence significative. Les internes seniors ont été significativement meilleurs concernant les problèmes de consultation et la majorité d'entre eux ont obtenu une note supérieure à la moyenne totale du groupe. Malgré notre manque d'expérience et les difficultés logistiques, nous n'avons pas rencontré de difficulté dans le développement et l'organisation de l'examen. Tous les participants ont confirmé que les situations cliniques présentées étaient réalistes et que les patients simulés étaient crédibles. Conclusions: Les résultats de cette expérience pilote d'ECOS en urologie prouvent la faisabilité d'un tel instrument pour évaluer la performance des internes et les déficiences éventuelles de l'enseignement, notamment l'activité de consultation. Cette expérience doit être développée afin d'explorer ses qualités psychométriques.

Mots clés ECOS, patients standardisés, urologie, internes.

Summary Background: Objective Clinical Examination (OSCE) is considered as the gold standard for evaluating clinical competence. Experience with OSCE remains limited in France. Objective: To assess the feasibility and acceptability of OSCE for the evaluation urology residents. Material and Methods: 20 urology residents went through a circuit of 5 standardised patients-based OSCE stations of 10 minutes duration. The selection of problems was based on educational objectives of urology residency programs. Written questionnaires with Likert-type scales were used to measure OSCE acceptability. Student $t$ test was used to compare the performance between junior and senior residents. Results: Globally, senior residents performed better, but without significant differences. Senior resident scores on outpatient problems were significantly higher. A majority of senior residents had higher scores than the mean score of all residents. Despite the lack of experience and the logistic difficulties, we were able to develop and organise this examination. All the participants agreed that the clinical situations were realistic and that the simulated patients were credible. Most participants agreed that the cases sampled were representative of urology practice. Conclusions: The results of this first reported OSCE applied to urology residency support the feasibility of the procedure in assessing the performance of urology residents. These results raise questions regarding the training of outpatient consultation skills to residents. This experiment warrants further series to explore the instrument psychometric features.
\end{abstract}

Key Words OSCE, standardized patient, urology, resident.

Pedagogie Medicale 2000; $1: 33-39$.

*Service d'urologie - CHU Rouen - Hôpital Charles Nicolle - Rouen, France - **Centre de Pédagogie en Sciences de la Santé (CPSS) - Faculté de Médecine - Sherbrooke - Canada ** * Unité de Recherche et Développement en Education des Sciences de la Santé (URDESS) - Faculté de médecine - Université de Montréal - Montréal - Canada - Correspondance et tirés à part : Dr Louis Sibert - Service d'urologie - CHU Rouen - Hôpital Charles Nicolle - 1 rue de Germont - 76031 Rouen Cédex - Tel. : 0232888173 - Fax. : 0232888205 - Courrier électronique : louis.sibert@chu-rouen.fr 


\section{Introduction}

L'urologie est une spécialité médico-chirurgicale. Au cours de leur cursus, les internes en urologie doivent acquérir, outre les habiletés techniques opératoires, des habiletés de recueil de données, relationnelles, de résolution de problèmes, diagnostiques et thérapeutiques, l'ensemble constituant la compétence clinique ${ }^{1}$. Actuellement, le mode d'évaluation des internes est très variable d'une région à l'autre et est généralement basée sur des examens soit subjectifs (examens oraux portant sur un seul cas clinique), soit ne testant qu'une seule dimension de la compétence clinique : les connaissances théoriques factuelles (examen écrit par QCM). En France, tout comme ailleurs, l'évaluation de la performance et de la formation des urologues se doit d'être de grande qualité afin d'assurer la crédibilité de la profession et du processus de délivrance du diplôme. L'évaluation de la compétence clinique doit tenir compte de son caractère multidimensionnel.

Développé par Harden ${ }^{2}$, il y a plus de vingt ans, l'Examen Clinique Objectif Structuré (ECOS) est considéré comme l'instrument se rapprochant le plus de l'évaluation idéale de la compétence clinique ${ }^{3}$. Un examen ECOS est composé d'une séquence de situations cliniques présentées dans différentes "stations" de quelques minutes chacune, constituant un " circuit " à travers lequel les candidats font la rotation. Les candi- dats interagissent avec un patient simulé et standardisé et doivent compléter une série de tâches prédéterminées. Leur performance est évaluée par un observateur-examinateur qui utilise une grille d'observation spécifique au contenu de la situation et aux tâches demandées. L'expérience de ce type d'examen est encore très limitée en France, a fortiori au niveau de l'internat et des spécialités chirurgicales. Dans le cadre de sa réforme pédagogique, la Faculté de Médecine de Rouen introduit progressivement ce type d'évaluation dans son cursus. Plusieurs expériences d'ECOS ont été testées en formatif au niveau du second cycle. Un projet d'ECOS sommatif destiné au $3^{\text {e }}$ cycle de Médecine Générale est en cours de développement. Dans ce contexte, nous rapportons le développement et l'implantation de la première expérience française d'évaluation formative des internes en urologie par ECOS.

\section{Développement}

Le développement de cet ECOS a été effectué par un groupe composé par 2 urologues universitaires et 2 libéraux, qui avaient tous au moins 10 ans de pratique clinique. Le respect de la méthodologie de rédaction des cas, des scénarios, des grilles d'observations, des instructions aux patients et aux observateurs était sous la responsabilité d'un des membres du groupe qui avait acquis une expertise en rédaction de cas ECOS ${ }^{4}$. La

\begin{tabular}{|c|c|c|}
\hline \multicolumn{3}{|c|}{ Tableau 1 : ECOS en urologie : liste des stations } \\
\hline Problèmes cliniques & Contexte & Objectifs d'évaluation \\
\hline 1. Impuissance & Consultation & Interrogatoire, investigations, communication, organisation \\
\hline $\begin{array}{l}\text { 2. Rétention d'urine fébrile } \\
\text { avec prostatite }\end{array}$ & Urgence & $\begin{array}{l}\text { Interrogatoire, investigations, traitement, } \\
\text { communication, organisation }\end{array}$ \\
\hline $\begin{array}{l}\text { 3. Incontinence urinaire } \\
\text { à l'effort }\end{array}$ & Consultation & $\begin{array}{l}\text { Interrogatoire, investigations, communication, } \\
\text { organisation }\end{array}$ \\
\hline 4. Elévation isolée des PSA & Consultation & $\begin{array}{l}\text { Interrogatoire, investigations, traitement, communication } \\
\text { organisation }\end{array}$ \\
\hline $\begin{array}{l}\text { 5. Colique néphrétique } \\
\text { lithiasique et grossesse }\end{array}$ & Urgence & $\begin{array}{l}\text { Interrogatoire, investigations } \\
\text { traitement, communication, organisation }\end{array}$ \\
\hline
\end{tabular}




\section{Tableau 2 : grille d'observation « Incontinence urinaire "}

Instructions reçues par le candidat : Madame B..., 53 ans, vient vous consulter car elle se plaint de fuites d'urine. Vous avez 10 minutes pour réaliser un interrogatoire pertinent

le candidat sinforme sur

1.0 Les caractéristiques des fuites

Fait (cocher)

1.1 Date de début de l'incontinence

1.2 Facteur déclenchant (effort)

1.3 Impériosités

O

$O$

1.4 Si impériosités :

1.4a) Délai de continence?

1.4b) fréquence?

O

\subsection{L'importance des fuites}

2.1 Fréquence des fuites

2.2 Intensité de l'effort déclenchant

2.3 Port de garniture

2.4 Combien et sont-elles toujours mouillées?

$O$

O

3.0 Les troubles associés

3.1 Infections urinaires

3.2 Hématurie

$O$

3.3 Dysurie

3.4 Troubles $d u$ transit

$O$

3.5 Dyspareunie

$O$

O

4.1 Sur les activités physiques

4.2 Sur les activités professionnelles et sociales

4.3 Sur l'humeur et l'état psychologique

O

$O$

\subsection{Les antécédents}

5.1 Obstétricaux

$O$

5.2 Chirurgicaux et médicaux

5.3 Traitements antérieurs des fuites

5.4 Prises médicamenteuses

5.5 Poids et taille

$O$

$O$

6.0 propose un examen physique

\subsection{Communication}

7.1 Communication claire

7.2 Sans jargon médical

7.3 Langage non verbal

Pauvre $->$ excellent

$\begin{array}{lllllll}0 & 2 & 4 & 6 & 8 & 10\end{array}$

$\begin{array}{llllll}O & O & O & O & O & O\end{array}$

$\begin{array}{llllll}O & O & O & O & O & O\end{array}$

$\begin{array}{llllll}O & O & O & O & O & O\end{array}$

\subsection{Organisation de l'interrogatoire}


rédaction des cas a débuté 5 mois avant l'examen et a suivi les stratégies de mise au point d'évaluation de la compétence clinique selon des étapes clairement délimitées $^{4,6}$. Le Choix de cas cliniques a été réalisé en fonction de leur représentativité de la pratique urologique (Tableau 1). Pour chaque cas clinique ont été identifiés le mode de rencontre (urgence ou consultation), le symptôme de présentation, le diagnostic sous-jacent, l'âge et le sexe du patient. La répartition de ces items visait à refléter l'activité urologique adulte habituelle. Le choix des composantes de la compétence clinique à évaluer a tenu compte du fait que les candidats étaient des internes de spécialité ; les stratégies d'investigation et de prise en charge thérapeutique ont été privilégiées, l'interrogatoire et le recueil de données ont été plus développés que l'examen physique, il n'y avait pas d'évaluation des habiletés techniques. Le tableau 2 illustre la grille d'observation utilisée pour un des 2 cas de consultation. Cette démarche planifiée et structurée d'évaluation obéissant à un canevas préétablis précis a grandement facilité l'aboutissement de ce projet d'évaluation malgré le manque d'expérience des organisateurs ${ }^{7}$.

L'entraînement des patients simulés a commencé 1 mois avant l'examen avec l'aide d'infirmières instructrices. Sept patients simulés ( 3 hommes, 2 femmes, 2 remplaçants) ont été recrutés à partir d'un groupe de patients simulés formés dans le cadre du programme d'évaluation du 3 cycle de Médecine Générale de la Faculté. Ce groupe de patients simulés était constitué de personnes sans relation avec l'hôpital, la médecine ou l'enseignement.

Les observateurs ont été recrutés parmi les responsables de l'enseignement de la discipline. Leur formation a consisté à leur envoyer 3 semaines avant l'examen la grille d'observation et les instructions pour qu'ils aient suffisamment de temps pour se les approprier. Ils ont été ensuite plusieurs fois personnellement contactés afin de vérifier la pertinence des grilles et leur bonne compréhension du déroulement de l'examen. Tous les observateurs ont reçu exactement les mêmes consignes et ont été réunis brièvement 15 minutes avant l'examen afin d'uniformiser au maximum l'observance des grilles.

\section{Organisation}

Les candidats étaient 20 internes inscrits au diplôme d'étude spécialisée en urologie des 4 facultés de médecine de la région Nord (Amiens, Caen, Lille et Rouen). Tous ont accepté de participer volontairement à ce test.
Il y avait 8 internes juniors ( $1^{\mathrm{e}}$ et $2^{\mathrm{e}}$ année) et 12 internes seniors $\left(3^{\mathrm{e}}, 4^{\mathrm{e}}\right.$ et $5^{\mathrm{e}}$ année d'internat). L'examen $s^{\prime}$ est déroulé sur 1 jour et a consisté en un circuit de 5 stations avec patient simulé de 10 minutes chacune. La durée du circuit était de 54 minutes par candidat 5 stations de 10 minutes avec 1 minute de repos entre chaque station). Le même circuit a été répété 4 fois pour pouvoir faire passer tous les internes, 2 fois le matin, 2 fois l'après midi. 5 internes étaient testés par circuit. Les internes ont été répartis au hasard dans chaque circuit. A la fin de l'examen, tous les candidats et les observateurs ont rempli un questionnaire écrit type Likert contenant 6 questions et 4 catégories (tout à fait en désaccord, partiellement en désaccord, partiellement d'accord, tout à fait d'accord) pour évaluer leurs opinions sur le déroulement de l'examen et son contenu. Ce type de questionnaire a été utilisé et validé par des travaux antérieurs ${ }^{8}$. Chaque station était notée sur 100 avec la même pondération. La note de chaque candidat correspondait à la somme des scores obtenus à chaque cas divisée par le nombre de cas.

L'objet de notre examen était de tester la faisabilité de l'ECOS, tant au niveau de son développement que de son système de notation. Notre but n'était pas d'évaluer les candidats, mais plus " humblement ", de voir si notre première expérience dans ce cadre précis, restait cohérente avec le niveau supposé des internes en fonction de leur ancienneté dans le cursus. Les candidats ont été informés clairement de ce choix arbitraire avant l'examen. Dans cet ordre d'idée, nous avons effectué une comparaison statistique des performances des internes juniors et seniors par un test $\mathrm{t}$ de Student.

\section{Résultats}

Le tableau 3 résume les résultats chiffrés obtenus. La performance globale des internes seniors était légèrement supérieure à celles des juniors (seniors : 51,3 \pm 7,8; juniors $45 \pm 5,1 ; p=0,09)$. Les internes seniors ont réussi une meilleure performance aux problèmes de consultation que les juniors $(54,9 \pm 7,4$ versus $46,9 \pm 6,4$; $\mathrm{p}=0,04)$ et un plus grand nombre d'entre eux ont dépassé la note de passage (9 sur 12 contre seulement 2 sur 8 juniors).

Le taux de réponse à notre questionnaire a été de $100 \%$ (Tableau 4). Dans la présentation des résultats, les participants partiellement en accord et tout à fait d'accord avec une question ont été groupés ensemble. L'immense majorité de participants étaient d'accord pour penser 


\section{Tableau 3 : comparaison des performances des internes juniors et seniors}

\begin{tabular}{|c|c|c|c|}
\hline Résultas & $\begin{array}{l}\text { Internes } \\
\text { Juniors }(\mathrm{n}=\mathbf{8})\end{array}$ & $\begin{array}{l}\text { Internes } \\
\text { seniors }(n=12)\end{array}$ & $\begin{array}{l}\text { Différence significative } \\
\text { (test } \mathbf{t} \text { de Student) }\end{array}$ \\
\hline $\begin{array}{l}\text { Score global } \\
\text { - moyenne } \\
\text { - D.S. }\end{array}$ & $\begin{array}{l}45 \\
5,1\end{array}$ & $\begin{array}{c}51,3 \\
7,8\end{array}$ & $N S(p=0,09)$ \\
\hline $\begin{array}{l}\text { Problèmes de consultation } \\
\text { - moyenne } \\
\text { - D.S. }\end{array}$ & $\begin{array}{l}46,9 \\
6,4\end{array}$ & $\begin{array}{l}54,9 \\
6,4\end{array}$ & $S(p=0,04)$ \\
\hline $\begin{array}{l}\text { Problèmes d'urgence } \\
\text { - moyenne } \\
\text { - D.S. }\end{array}$ & $\begin{array}{l}42,1 \\
2,3\end{array}$ & $\begin{array}{l}42,8 \\
7,4\end{array}$ & $N S(p=0,11)$ \\
\hline $\begin{array}{l}\text { Nombre de candidat } \\
\text { avec note }>\text { note de passage }\end{array}$ & $2 / 8$ & $9 / 12$ & \\
\hline $\begin{array}{l}\text { Chaque station est notée su } \\
\text { Le score total par candidat } \\
D S: \text { déviation standard } \\
N S: \text { pas de différence signi }\end{array}$ & $\begin{array}{l}\text { la somme des sco } \\
\text { ye }(p>0.05)\end{array}$ & à chaque cas divis & nombre de cas \\
\hline
\end{tabular}

que l'échantillonnage de cas présentés était représentatif de la pratique urologique, que la durée des stations était adéquate, et que, globalement, hormis les compétences techniques, ce type d'examen pouvait évaluer les compétences nécessaires à la pratique correcte de l'urologie. Surtout, bien qu'il s'agissait d'une première tentative, aussi bien pour les organisateurs que pour les patients simulés et leurs instructrices, tous les candidats et tous les observateurs ont estimé que les situations cliniques présentées étaient réalistes et que les patients simulés avaient été crédibles. Nous avons ensuite demandé aux candidats s'ils avaient déjà rencontré au cours de leur pratique, les problèmes proposés lors de ce test. Les situations d'urgence (rétention d'urine avec prostatite, colique néphrétique et grossesse) étaient familières à plus de trois quarts des internes.

En revanche, nous avons constaté que les deux tiers des internes, y compris les seniors n'avaient jamais rencontré, au cours de leur pratique, les problèmes de consultations élaborés pour cet examen. La prise en charge de l'incontinence urinaire féminine et des troubles érectiles sont pourtant des objectifs d'apprentissage important des programmes d'enseignement d'urologie et sont des problèmes très fréquemment rencontrés au cours de la pratique urologique.

\section{Discussion}

Une évaluation par ECOS n' à ce jour jamais été publiée dans le domaine de l'urologie. Nous sommes bien conscients des limites de cette expérience. Le manque d'habitude des participants à ce type d'examen, le trop petit nombre de candidats, doivent rendre prudent dans l'interprétation des résultats. De même, le trop petit nombre de stations ne permet pas d'obtenir une bonne validité de contenu, même si certaines expériences d'ECOS en chirurgie avec un nombre également limité de stations ont pu obtenir un coefficient de fiabilité acceptable ${ }^{9}$. Le système de notation adopté est arbitraire et ne peut en aucun cas être utilisé pour établir un véritable classement ou pour juger de façon précise la com- 


\section{Concepts et Innovations}

pétence réelle de chaque candidat. Le principal objectif de cette étude n'était cependant pas d'obtenir des différences statistiques mais d'évaluer la faisabilité de ce mode d'évaluation au sein de notre milieu.

Malgré toutes ses imperfections, cette expérience nous a apporté des renseignements intéressants concernant ce groupe d'internes et leur formation. En dépit du manque de validité de notre système de notation, nous avons pu déceler certaines différences de performance parmi les internes, qui pourraient être cohérentes avec leurs niveaux de formation. La note moyenne des internes seniors est meilleure que celle des juniors. De plus, les internes seniors semblent avoir été plus performants sur les 2 cas de consultation que les juniors. La grande variabilité des notes obtenues d'un cas à l'autre peuvent probablement être expliquées par le biais dans la notation et confirment la nécessité d'un plus grand nombre de cas pour une évaluation correcte de la compétence clinique $^{10}$. Nous interprétons le fait que les internes n'étaient pas habitués aux problèmes de consultation, comme un reflet probable du manque d'enseignement spécifique des compétences de consultant en urologie. Malgré leur nombre limité, les situations choisies sont en effet pertinentes à la pratique.

L'ensemble des participants semble avoir apprécier l'expérience et a témoigné une opinion favorable au déve- loppement de ce type d'évaluation formative. Notre première expérience d'utilisation de patients simulés a été très favorablement accueillie, comme le prouve les résultats de notre questionnaire (Tableau 4). Outre son acceptabilité, la faisabilité de l'expérience nous semble démontré. Malgré les limites logistiques habituelles des ECOS, nous n'avons pas rencontré de difficultés majeures dans le développement, l'implantation de l'examen, ni dans l'utilisation de patients simulés. De plus, le coût par candidat (152.20 Euros) est comparable a ceux d'études déjà publiées ${ }^{11}$.

A notre sens, l'un des intérêts de cet ECOS, est qu'il a rappelé à nos internes que des compétences sémiologiques de base, telle que l'interrogatoire, l'écoute du patient, la façon de se comporter et de s'exprimer pouvaient faire l'objet d'évaluation. Comme dans toutes les disciplines chirurgicales, les internes en urologie s'investissent beaucoup dans l'acquisition des habilités techniques, diagnostiques et thérapeutiques au détriment, parfois, des compétences d'interrogatoire, d'examen physique et relationnel. Il est bien connu que tout étudiant adapte son apprentissage au type d'évaluation auquel il est soumis. Si nous arrivons a répéter ce genre d'examen, les internes pourraient modifier leurs stratégies d'apprentissage. Ces premiers résultats ont également entrâné une prise de conscience de la part des

Tableau 4 : opinions des participants sur l'ECOS en urologie

\begin{tabular}{|l|c|c|}
\hline Affirmations & $\begin{array}{c}\text { \% de candidats } \\
\text { d'accord (n = 20) }\end{array}$ & $\begin{array}{c}\text { \% d'observateurs } \\
\text { d'accord (n = 10) }\end{array}$ \\
\hline $\begin{array}{l}\text { - L'échantillonnage de cas utilisé est } \\
\text { représentatif de l'activité urologique }\end{array}$ & $84 \%$ & $90 \%$ \\
- Les patients simulés étaient crédibles & $100 \%$ & $100 \%$ \\
- Les situations cliniques présentées étaient réalistes & $100 \%$ & $100 \%$ \\
- La durée des stations était adéquate & $84 \%$ & $88 \%$ \\
- L'ECOS permet de mesurer globalement les \\
compétences d'un urologue (habiletés techniques exclues)
\end{tabular}


enseignants d'urologie des déficits de l'enseignement de la consultation, qui est pourtant un aspect majeur de la pratique quotidienne en urologie ${ }^{12}$. Nous avons institué une consultation quotidienne tenue par les internes, un guide avec référentiels et arbres décisionnels a été rédigé et distribué aux internes, des réunions de présentation de dossier de consultation sont régulièrement organisées. Nous avons suscité une plus large réflexion multi- disciplinaire sur les compétences de consultant ${ }^{13}$. Malgré ses limites, cette étude pilote a prouvé que l'ECOS pouvait être un outil pédagogique et d'évaluation formative utilisable pour l'enseignement des internes d'urologie. Des études complémentaires sont bien entendues nécessaires pour juger de ses qualités psychométriques.

\section{Références}

1. Norman G. Defining competence: A methodological review. In Neufeld $V$. and Norman $G$. editors. Assessing clinical competence, New-York : Springer, 1985.

2. Harden R, Stevenson M, Downie W, Wilson G. Assessment of clinical competence using objective structured examination. BMJ $1975 ; 1: 447-451$.

3. Van Der Vleuten CP. Assessment of clinical skills with standardized patients : state of the art. Teaching and Learning in Medicine 1990 ; 2 : 58-76.

4. Sibert L, Grand'Maison P, Charlin B, Grise P. Evaluation de la compétence clinique en urologie: approche innovatrice basée sur l'observation de la compétence. Progrès en urologie 1997 ; 581-589.

5. Newble D I, Dawson B, Dauphinee D. Guidelines for assessing clinical competence. Teaching and Learning in Medicine 1994; 6 : 213-220.

6. Sloan D A, Donnelly $M B$, Schwartz $R W$, Strodel $W E$. The objective structured clinical examination. The new gold standard for evaluating postgraduate clinical performance. An Surg 1995; 222 : 735742.

7. Marshall K. Comment rédiger une épreuve ECOS. Québec(Canada) : CESSUL, 1994.
8. Grand'Maison P, Brailovsky C A, Lescop J. Content validity of the Quebec licensing examination (OSCE). Canadian Family Physician 1996 ; 42 :254-259.

9. Sloan D A, Donnelly $M$ B, Johnson S B, Schwartz $R W$, Strodel WE. Assessing surgical resident's and medical student's interpersonal skills. Journal of Surgical Research 1994; 57 : 613-618.

10. Newble D I, Swanson D B. Psychometric characteristics of the Objective Structured Clinical Examination. Medi Ed 1998; 22 : 325-334.

11. Cusimano M D, Cohen R, Tucker W, Murnaghan J, Kodama R, Reznick R. A comparative analysis of the coast of administration of an OSCE. Acad Med 1994; 69 : 76-81.

12. Sibert L, Charlin B, Lachkar A, Bugel H., Grise $P$. Les compétences de consultants en urologie: Une modélisation pour l'enseignement et l'évaluation. Progrès en Urologie, sous presse 2000.

13. Sibert L, Charlin B, Lachkar A, Grise P. A qualitative analysis to define learning and assessment objectives of consultation skills in a speciality residency programme. Med Teach 2000 accepté pour publication. 